\title{
Potential Uses of the Differential Propagation Phase Constant to Estimate Raindrop and Hailstone Size Distributions
}

\author{
ILANA STEINHORN, MEMBER, IEEE, AND DUSAN S. ZRNIC', SENIOR MEMBER, IEEE
}

\begin{abstract}
One of the Doppler radars operated by the National Severe Storms Laboratory has a new capability to measure the difference between propagation phase constants at horizontal and vertical polarization. This study examines the uses of this parameter $K_{D P}$ in addition to the reflectivity factor $Z_{H}$ and the differential reflectivity $Z_{D R}$, to obtain new information about rain and hail. It is shown from theory and experiments that a third parameter of the drop size distribution, obtained from $K_{D P}$, may be used to support $Z_{D R}$ measurements, and/or to point out mixed phase hydrometeors. Quantitative information on hail size distribution may be obtained for small-size hailstones in cases when their major axes are nearly vertically aligned, giving rise to negative $Z_{D R}$ and $K_{D P}$ values.
\end{abstract}

Keywords-Differential propagation phase, drop size distribution, hail size distribution, remote sensing of hail.

\section{INTRODUCTION}

$\mathrm{D}$ OPPLER RADAR capable of transmitting and receiving horizontally and vertically polarized fields measures the reflectivity factors $Z_{H}$ and $Z_{V}$ at two orthogonal polarizations. Their ratio, the differential reflectivity $Z_{D R}$, is related to shape and degree of common orientation of the precipitating hydrometeors. Raindrops are oblate and form a highly oriented medium, in which each drop's shape is uniquely related to size; therefore, with two reflectivity factors, both parameters of a two-parameter drop size distribution model may be calculated, and used for a better rain rate estimation [1], [2]. Also, due to the difference in shape and falling behavior between raindrops and hailstones, considerable success in hail detection has been documented [3]. This was achieved by noting that $Z_{D R}$ values in hail regimes are low, close to zero, while the reflectivity is relatively high. Additional information on rain and hail may be determined from measurements of the propagation phase constants at horizontal and vertical polarizations. The differential propagation phase constant $K_{D P}$ is an independent quantity which, like $Z_{D R}$, is a measure of the hydrometeors' shape and orientation. Although there have been no studies concerning the behavior of $K_{D P}$ through hail regions, it has been shown that

Manuscript received October 19, 1987; revised April 19, 1988. This work was supported in part by the Joint Systems Program Office of NEXRAD.

The authors are with NOAA, Environmental Research Laboratories, National Severe Storms Laboratory, Norman, OK 73069.

IEEE Log Number 8821912. the rainfall rate is correlated with the differential phase constant [4], or alternately, that $K_{D P}$ may be related to the liquid water content and the mass weighted mean axis ratio [5].

Experimental study of $K_{D P}$ properties in rainfall and hail regimes is now possible with the National Severe Storms Laboratory's (NSSL) dual-polarized Doppler radar. Analysis of the statistical error in $K_{D P}$ estimates has been done by Sachidananda and Zrnic' [6], who also showed theoretically that the direct relationship between differential propagation phase constant $K_{D P}$ and the rain rate $R$ was less sensitive to drop size distribution (DSD) variations than the reflectivity factor versus $R$ relationship. They suggested that $K_{D P}$ could replace $Z$ in rain rate estimation. Analysis of some data sets showed [7] that $K_{D P}$ measurements were generally consistent with measurements of reflectivity $Z_{H}$ and differential reflectivity $Z_{D R}$. However, the expected accuracy of inferred rain rate was not achieved at low rain rates, presumably because of signal contamination through antenna sidelobes [7]. We show here that the observed scatter of $K_{D P}$ at medium and higher reflectivities is likely due to physical causes. Part of the error in rain rate estimation is from mixed-phase particles that would be interpreted as raindrops, therefore, improvement is possible by identifying these.

It is suggested that a third measured parameter $K_{D P}$ may be used to solve for a third parameter of a three parameter drop size distribution (DSD) model, which may lead to a better rain rate estimation, whereas its dependency on the orientation of the hydrometeors may lead to better hail discrimination. The purpose of this study then is to examine how $K_{D P}$ measurements may add information about rain characteristics and hail identification, when used in conjunction with reflectivity and differential reflectivity. Theoretical considerations are based on the assumption of Rayleigh scattering at a wavelength of $10 \mathrm{~cm}$, and radar data of rain and hail storms are analyzed and compared with theory.

\section{Computation of Polarization Parameters}

The phase of the backscattered electric field depends on the hydrometeors' size, shape, and quantity, their distance from the radar, and the polarization of the signals. Differential phase shift is experienced in cases of non- 
spherical particles with a preferred orientation. Based on Van de Hulst's method [8], the complex phase constant at vertical or horizontal polarization may be calculated, assuming a particular shape and size distribution of the hydrometeors. It is assumed that the hydrometeors may be modeled as oblate spheroids. This assumption has been supported by observations of raindrops, and to a lesser extent, is reasonable for hailstones [9].

The horizontal and vertical complex propagation constants of the medium $K_{H, V}$ may be expressed as [10]

$$
K_{H, V}=k_{0} \int_{0}^{D_{m}} Q_{H, V}(e, m) D^{3} N(D) d D
$$

where $D$ is the diameter of a sphere with volume equal to that of the spheroid, $a$ and $b$ are the semi-minor and semimajor axes, $N(D)$ is the drop size distribution, $D_{m}$ is the maximum diameter of scatterers, and $k_{0}$ is the free space propagation constant. The eccentricity $e$ is a function of the axis ratio, $a / b, e^{2}=1-a^{2} / b^{2}$. It is further assumed that the spheroidal raindrops have zero canting angle, whereas spheroidal hailstones are canted at $90^{\circ}$ (i.e., their minor axis is horizontally oriented). With the assumption of Rayleigh scattering, $Q_{H, V}$ are functions of the eccentricity $e$ and refractivity $m$ of the hydrometeors as follows [10]:

$$
Q_{H, V}=\frac{\pi\left(m^{2}-1\right)}{12\left[1+\left(m^{2}-1\right) A_{H, V}\right]}
$$

where

$$
\begin{aligned}
A_{H}= & \frac{1-e^{2}}{2 e^{2}}\left\{\left(1-e^{2}\right)^{-1 / 2} e^{-1}\right. \\
& \left.\cdot \tan ^{-1}\left[e /\left(1-e^{2}\right)^{1 / 2}\right]-1\right\} \\
A_{V}= & 1-2 A_{H} .
\end{aligned}
$$

The two-way differential propagation phase constant $K_{D P}$ is defined as the real part of the difference between the horizontal and vertical complex propagation constants [10]

$$
K_{D P}=\operatorname{Re}\left(K_{H}-K_{V}\right)(360 / \pi)\left(\operatorname{deg} \mathrm{m}^{-1}\right) .
$$

The reflectivity factors at horizontal and vertical polarizations $Z_{H}$ and $Z_{V}$ are defined by [11]

$$
Z_{H, V}\left(\mathrm{~mm}^{6} \mathrm{~m}^{-3}\right)=\frac{1}{|K|^{2}} \int_{0}^{D_{m}} S_{H, V}(e, m) D^{6} N(D) d D
$$

where $S_{H, V}$ are functions of the eccentricity and refractivity of the drops or the hailstones

$$
S_{H, V}=\frac{16 \pi^{2}\left|m^{2}-1\right|^{2}}{9\left|4 \pi+\left(m^{2}-1\right) P_{H, V}\right|^{2}}
$$

where

$$
\begin{aligned}
& P_{V}=\left(4 \pi / e^{2}\right)\left\{1-\left[\left(1-e^{2}\right) / e^{2}\right]^{1 / 2} \sin ^{-1} e\right\} \\
& P_{H}=\left(4 \pi-P_{V}\right) / 2 ;|K|^{2}=\left|m^{2}-1\right| /\left|m^{2}+2\right| .
\end{aligned}
$$

The differential reflectivity $Z_{D R}$ (in $\mathrm{dB}$ ) between these two reflectivity factors for horizontal and vertical polarizations is [1]

$$
Z_{D R}=10 \log _{10}\left(Z_{H} / Z_{V}\right)
$$

Both $K_{D P}$ and $Z_{D R}$ depend on the anisotropy of the medium, and have positive values in rain. Also, they depend on the integral of drop size distribution, with different weighting functions; the integrand of $K_{D P}$ is a function of $D^{3}$ compared to the $D^{6}$ dependence of $Z_{H}$ and $Z_{V}$. Furthermore, $K_{D P}$ is obtained from the difference, whereas $Z_{D R}$ is obtained from the ratio of such integrals.

$Z_{D R}$ from time series data is calculated using square law power estimates [12]

$$
Z_{D R}(d B)=10 \log _{10}\left[\frac{1}{M} \sum_{i=1}^{M}\left|H_{2 i}\right|^{2} / \frac{1}{M} \sum_{i=1}^{M}\left|V_{2 i+1}^{2}\right|\right]
$$

where $H$ and $V$ are complex voltages corresponding to alternately received electric fields at a horizontal and vertical polarization. In our experiment, the number of samples $M$ was 256. Estimation of $K_{D P}$ is done as follows [6]:

$$
K_{D P}=\left[\phi_{D P}\left(r_{2}\right)-\phi_{D P}\left(r_{1}\right)\right] /\left[r_{2}-r_{1}\right]
$$

where $\phi_{D P}(r)$ is the phase shift at a range $r$ and is estimated, for each range, from $M$ samples

$$
\begin{aligned}
\phi_{D P} & =0.5 \arg \left[R_{a} R_{b}^{*}\right] \\
R_{a} & =(2 / M) \sum_{i=1}^{M / 2} H_{2 i}^{*} V_{2 i+1} \\
R_{b} & =(2 / M) \sum_{i=1}^{M / 2} V_{2 i+1}^{*} H_{2 i+2} .
\end{aligned}
$$

Note that whereas $Z_{D R}$ is measured within radar resolution volumes, $K_{D P}$ is computed along a path between two spaced resolution volumes. Also, in order to reduce the ambiguity resulting from occasional noisy information, $K_{D P}$ data (6) are averaged in range over 16 consecutive range cells as follows:

$$
K_{D P_{j}}=\left[\sum_{i=j+1}^{j+8} \phi_{D P_{i}}-\sum_{i=j-7}^{j} \phi_{D P_{i}}\right] /\left[r_{j+8}-r_{j}\right] .
$$

The separation $r_{2}-r_{1}$ over which $K_{D P}$ is calculated, is matched to the averaging interval, which varies from 1.2$2.4 \mathrm{~km}$.

\section{Drop Size Distribution}

For the purpose of interpreting dual-polarization radar measurements, raindrops may be modeled as oblate spheroids, with minor axes vertically oriented. The equations for $Z_{H}, Z_{D R}$, and $K_{D P}(1-4)$ include several free parameters, which in the case of raindrops are the DSD parameters only. The refractivity $m$, which for raindrops undergoes slight changes with temperature, may be assumed constant, and the axis ratio of the drops $a / b$ can be uniquely related to the equivolume diameter $D[13],[14]$. 
With these assumptions, relatively simple computations are needed to solve (2) and (4) for the parameters of a two parameter drop size distribution $N(D)$. A distribution frequently used is the exponential distribution $N(D)=$ $N_{0} e^{-\Lambda D}$. With only one free parameter $\Lambda$, the value of $N_{0}$ is chosen as $8000 \mathrm{~mm}^{-1} \mathrm{~m}^{-3}$ for the DSD of MarshallPalmer [15], and $\Lambda$ may be computed from the reflectivity factor to obtain the rain rate. But in practice an appropriate $Z-R$ relationship must be chosen for each storm. When both $Z_{H}$ and $Z_{D R}$ measurements are available, $N_{0}$, which can vary significantly even within a short period of time, may also be calculated [16]. As there is evidence that the number of drops with diameter smaller than $1 \mathrm{~mm}$ is depleted, it seems that a gamma distribution of the form $N(D)=N_{0} D^{\mu} e^{-\Lambda D}$, with $\mu$ between 2 and 3 , would fit observational results better [17]. Variations between empirical relations and theoretical calculations may also be reduced by truncating the DSD at an upper limit [18]. When three independent parameters are measured, there is a possibility of estimating a third parameter of a DSD, either the exponent $\mu$ of a gamma distribution, or the maximum diameter of the drops $D_{m}$. Alternately, two parameters of the DSD may be examined against the additional information provided by the third measured parameter, perhaps to increase the accuracy of the calculated values or to point out the presence of other types of hydrometeors in the medium.

Calculations of $Z_{H}, Z_{D R}$, and $K_{D P}$, based on (2)-(4), are presented in Figs. 1 and 2 for a radar wavelength of 10 $\mathrm{cm}$. Generally, $K_{D P}$ follows similar trends as $Z_{D R}$. This is because larger drops are more oblate; hence, horizontal fields (aligned with the major axis) experience more phase shift when high concentration of big drops is present. It should be noted here that the three measurables, $Z_{H}, Z_{D R}$, and $K_{D P}$, are independent. However, whenever the DSD can be characterized by an exponential distribution with two unknowns or a gamma distribution with known $\mu$, the variables $Z_{H}$ and $K_{D P}$ are no longer independent. In such situations, $K_{D P}$ may be expressed in terms of $Z_{H}$ as $K_{D P}$ $=\alpha Z_{H}^{\beta} N_{0}^{1-\beta}$, where $\alpha$ and $\beta$ are functions of $D_{m}$ and $\Lambda$. In the case of exponential DSD with $D_{m}=8 \mathrm{~mm}$, these values are: $\alpha=4 \times 10^{-5}$ and $\beta=0.86$, and when $\mu=$ 3 they are $3 \times 10^{-5}$ and 0.88 , respectively; $K_{D P}$ is in deg $\mathrm{km}^{-1}, Z_{H}$ in $\mathrm{dBZ}$, and $N_{0}$ in $\mathrm{mm}^{-1} \mathrm{~m}^{-3}$. Therefore, measurements of $Z_{H}, Z_{D R}$, and $K_{D P}$ could play a significant role, either when the DSD cannot be characterized by less than three parameters, or when the precipitation medium contains mixed phase hydrometeors. Variations of $K_{D P}$ with $D_{m}$ (Fig. 1) are more pronounced when $Z_{D R}$ $>3 \mathrm{~dB}$ and $\mu$ is small, indicating the presence of big drops. Also, the sensitivity of $K_{D P}$ to variations in $\mu$ is larger than its sensitivity to variations in $D_{m}$ in the lower ranges of $Z_{D R}$, which is generally associated with small drops. If both $Z_{D R}$ and $Z_{H}$ are perfectly measured, the accuracy of $K_{D P}$ measurements required to distinguish between $D_{m}=5$ and $D_{m}=10 \mathrm{~mm}$, or between $\mu=0$ (exponential) and $\mu=3$, is about $5-10$ percent. Statistical analysis of $K_{D P}$ shows [6] that the standard error of $K_{D P}$

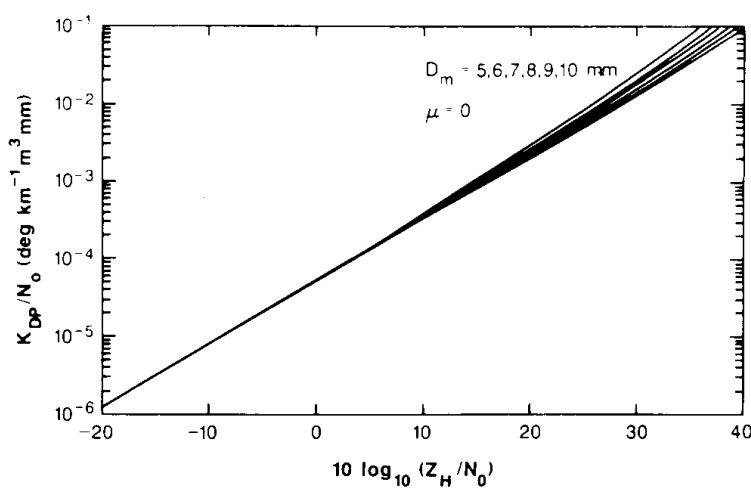

(a)

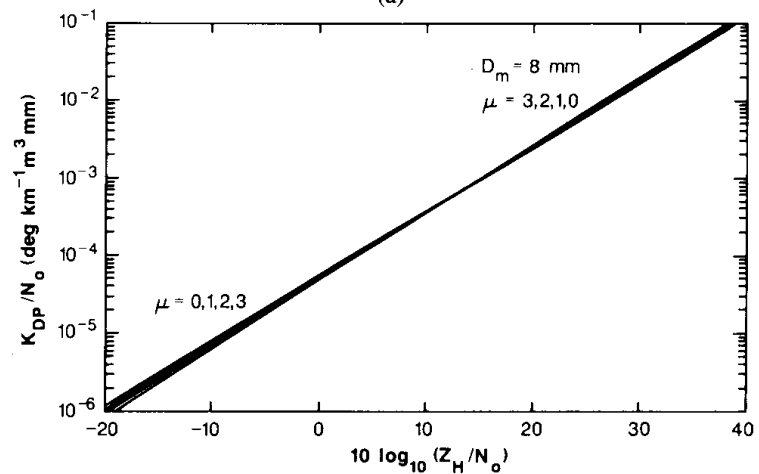

(b)

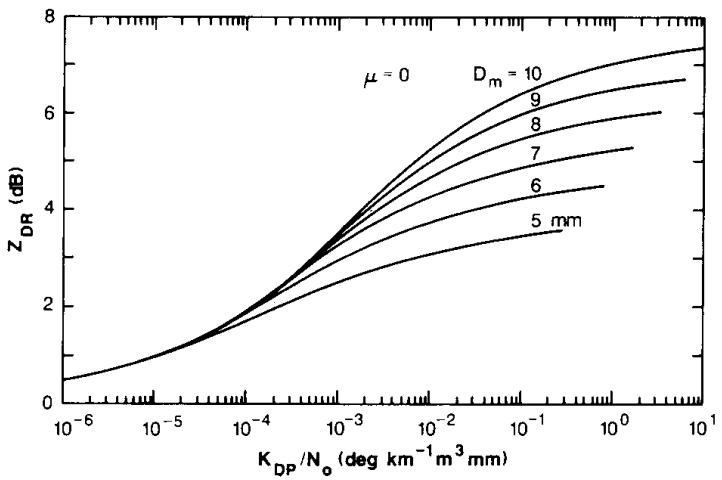

(c)

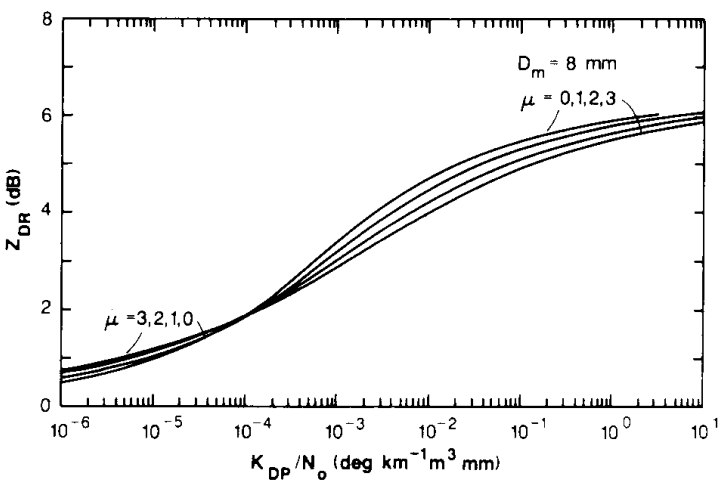

(d)

Fig. 1. Relationships between $K_{D P}, Z_{D R}, Z_{H}$, and DSD parameters for exponential and gamma distributions. Radar wavelength is $10 \mathrm{~cm}$, and in our convention $D^{\mu}$ is normalized to $\mathrm{mm}^{\mu}$ to have dimensions of $N_{0}$ invariant with $\mu$. 


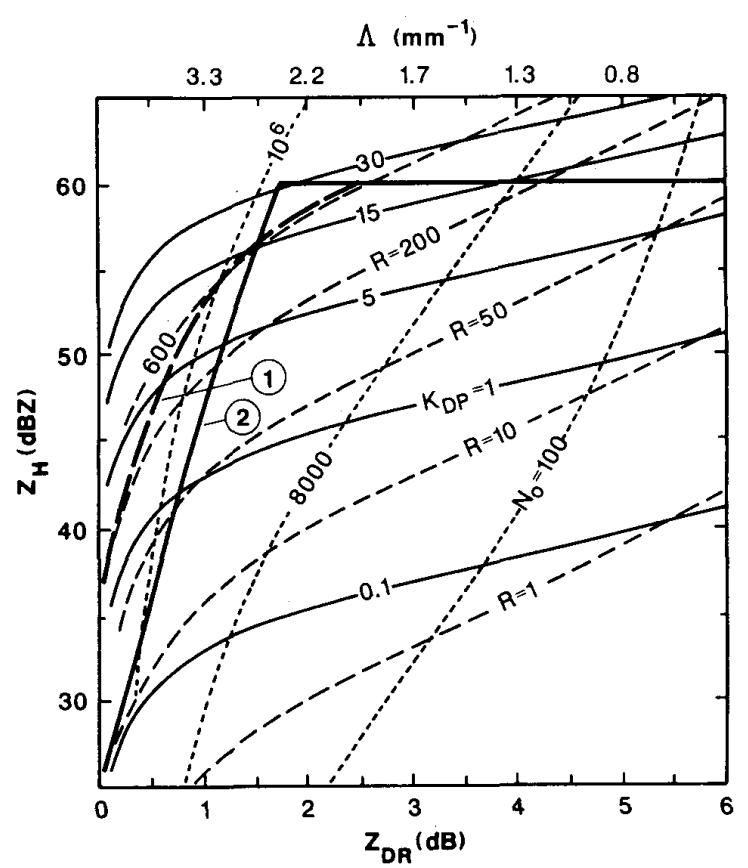

Fig. 2. Theoretical curves of $K_{D P}\left(\mathrm{deg} \mathrm{km}^{-1}\right), R\left(\mathrm{~mm} \mathrm{~h}^{-1}\right)$, and $N_{0}\left(\mathrm{~m}^{-3}\right.$ $\mathrm{mm}^{-1}$ ) in a $Z_{H}-Z_{D R}$ plane, for exponential DSD with $D_{m}=8 \mathrm{~mm}$. Rain boundaries, separating liquid from frozen hydrometeors, are 1) from [20] and 2) from [21].

measurements is of the order of $0.5 \mathrm{deg} \mathrm{km} \mathrm{km}^{-1}$. Therefore, if such an error is practically achievable, estimation of a third parameter of the DSD may be possible. If both $N_{0}$ and $\Lambda$ are accurately known, the addition of a third parameter would reduce the error in $R$ from 20 - 15 percent, as the contribution of variations in $\mu$ or $D_{m}$ to the rainfall rate is secondary to that due to changes in $\Lambda$ and $N_{0}[19]$. However, one may use $K_{D P}$ to increase confidence in the two parameters of the distribution, as $K_{D P}$ may replace $Z_{H}$ in the $Z_{H}-Z_{D R}$ method to achieve additional, independent evaluation of $N_{0}$, but with reduced spatial resolution.

In Fig. 2, curves of iso- $K_{D P}$ values, for exponential DSD with $D_{m}=8 \mathrm{~mm}$, are presented in a $Z_{H}-Z_{D R}$ plane. Also presented are $N_{0}$ and rain rate curves. High $Z_{H}$ generally indicates relatively high rainfall rate, associated with large drops (high $Z_{D R}$ values), or a high number of medium-size drops (medium $Z_{D R}$, high $N_{0}$ ). Although different types of rain may result in various $Z_{H}-Z_{D R}$ combinations, not all $Z_{H}-Z_{D R}$ combinations are physically possible; data from rain have a preferential region in a $Z_{H^{-}}$ $Z_{D R}$ plane. This has been confirmed from both radar measurements [20] and $Z_{H}-Z_{D R}$ computations based on disdrometer data [21]. The boundaries established by these two studies are in fairly good agreement; both have an upper $Z_{H}$ limit of $60 \mathrm{dBZ}$, but, as seen in Fig. 2, there are differences at lower values of $Z_{H}$ and $Z_{D R} . Z_{H}-Z_{D R}$ pairs on the boundary correspond to very high, unrealistic water contents that would seldom be present in nature. For instance, the calculated liquid water content for $Z_{H}=60$ $\mathrm{dBZ}$ and $Z_{D R}=1.3 \mathrm{~dB}$ is $100 \mathrm{~g} / \mathrm{kg} . Z_{D R}=2 \mathrm{~dB}$ would reduce the water content to a more reasonable, but still high, value of $50 \mathrm{~g} / \mathrm{kg}$. Lower $Z_{H}$ corresponds to lower water content for similar $Z_{D R}$ values; for instance, $Z_{H}=$ $50 \mathrm{dBZ}$ and $Z_{D R}=1.3 \mathrm{~dB}$ are produced by exponentially distributed raindrops with water content of $10 \mathrm{~g} / \mathrm{kg}$. High $Z_{H}$ and low $Z_{D R}$ are therefore signals for non-rain hydrometeors, presumably hail [21]. $K_{D P}$ within the rain boundary in Fig. 2 is between 0 and about $15 \mathrm{deg} \mathrm{km}{ }^{-1}$, and for pure rain must be consistent with $Z_{D R}$ and $Z_{H}$. When substantial inconsistencies among $K_{D P}, Z_{D R}$, and $Z_{H}$ occur, the ensemble of hydrometeors is less likely to be pure rain. This is a basis for possible discrimination among rain and other hydrometeors, using the three measured parameters.

\section{Hail Size Distribution}

Remote discrimination between hail and rain using dualpolarization measurements is based on the difference in shape, orientation, and size distribution between raindrops and hailstones. As noted previously, typical $Z_{D R}$ values in regions of rainfall range from $0.5-4 \mathrm{~dB}$. The larger $Z_{D R}$ generally corresponds to larger $Z_{H}$. However, radar measurements indicate that when hail is likely, $Z_{H}$ is high and $Z_{D R}$ is low [3]. This has led Aydin et al. [21] to define a function of $Z_{H}$ and $Z_{D R}$ that they call "hail signal," $H_{D R} . H_{D R}$ is the difference between the reflectivity $Z_{H}$ (for a given $Z_{D R}$ ) and the reflectivity for the same $Z_{D R}$ at a boundary between rain and hail (see Fig. 2). Dualpolarization measurements indicate $Z_{D R}$ values from hail are close to zero [3]. This is characteristic of relatively isotropic media. Zero $Z_{D R}$, together with high $Z_{H}$, are due to relatively symmetric or irregular but tumbling hailstones, with no mean preferred orientation. At present there is no conclusive experimental evidence regarding either the orientation of free-falling hailstones, or their mean shape; both may have large variability. Theory and measurements of falling hailstones suggest various orientations, one of which is random, due to tumbling [22], the other of which has aligned spheroids rotating about their minor axes, which is gyrating about the horizontal [23].

If hailstones could be modeled as oblate spheroids that fall with major axis vertically oriented (canting angle $90^{\circ}$ ), the scattering characteristics would lead to negative $Z_{D R}$ and $K_{D P}\left((1)\right.$ and (5)). In that case, $K_{D P}$ measurements would provide a means of obtaining a two-parameter hailstone size distribution, which shall be discussed next. Note that this applies to Rayleigh scattering, which at $S$ band frequencies occurs when hailstones have relatively small diameters $(<1 \mathrm{~cm})$. For large wet hail, Mie scattering may result in both positive and negative $Z_{D R}$ values [24]. Another assumption is that the axis ratio is independent of the size. This has been measured by Barge and Isaac [25] in Alberta, and Matson and Huggins [9] in Colorado, who report axis ratios with a narrow distribution between 0.73 and 0.85 . Knight [26] recently observed similarity of the shapes of small hailstones in Oklahoma and Alberta. Both exhibit axis ratios that have larger 
variability than previously reported; $b / a$ ranges from 0.95 (for 1- to 5-mm hail), to 0.7 (for 21- to 25-mm hail). The difference between experimental results may be attributed to natural variability of hailstones, and to some difference in experimental methods.

In our calculations we assume Rayleigh scattering, and oblate hailstones with canting angle of $90^{\circ}$ and eccentricity independent of size. Then $Z_{D R}$ becomes a function of axis ratio and refractive index only (3)-(5)

$$
Z_{D R}=10 \log _{10}\left(S_{H} / S_{V}\right) \text {. }
$$

It is possible to obtain a rough estimate of a two-parameter hail size distribution, as follows. From $Z_{D R}$ one can obtain the axis ratio $b / a$ for a known $m$, or alternately, $m$ for known $b / a$. From Equations (1), (2), and (4) one finds that $Z_{H}$ and $K_{D P}$ are related by

$$
\begin{aligned}
& Z_{H}\left[\mathrm{~mm}^{3}\right] / K_{D P}\left[\operatorname{deg~\mathrm {m}^{-1}]}\right. \\
& \quad=F_{1}(b / a, m) / F_{2}\left(\Lambda, D_{m}, \mu\right)
\end{aligned}
$$

where

$$
F_{1}=\pi S_{H} /\left[360 k_{0} \operatorname{Re}\left(Q_{H}-Q_{V}\right)\right]\left(m \mathrm{deg}^{-1}\right)
$$

and

$$
F_{2}=\int_{0}^{D_{m}} D^{3} N(D) d D \int_{0}^{D_{m}} D^{6} N(D) d D
$$

These relations are presented in Fig. 3 for dry, and 10percent wet hail of density $0.5 \mathrm{~g} / \mathrm{kg}$. The refractivity $m$ in $S_{H}$ was set to a value for liquid water, because that is used for automatic calculations of $Z_{H}$ in a calibrated radar.

Although the Rayleigh-Gunn theory of scattering should be used for sizes less than $1 \mathrm{~cm}$, we also show curves with $D_{m}$ of 2 and $3 \mathrm{~cm}$. For sizes up to $3 \mathrm{~cm}$, errors in $Z_{H}, Z_{D R}$, and $K_{D P}$ of individual scatterers are less than $1.5 \mathrm{~dB}, 0.4 \mathrm{~dB}$, and $3.4^{\circ}$; when sizes are integrated over drop size distributions the average errors are further reduced. Thus, presented curves are not strictly exact, but serve to illustrate general trends and provide quantitative results that have less uncertainty than radar measurements.

The hail size parameters are then estimated as follows: the axis ratio, and therefore $F_{1}$, are obtained from $Z_{D R}$ measurements, then $F_{2}$ may be calculated from $Z_{H}$ and $K_{D P}$ measurements to obtain $\Lambda$, using exponential or gamma DSD for an assumed, relatively small $D_{m}$. Once $\Lambda$ is known, $N_{0}$ may be calculated from measurements of either $Z_{H}$ (3) or $K_{D P}(1)$. This method may be used in the case of small hail, when both $Z_{D R}$ and $K_{D P}$ measurements yield negative values. The assumption that axis ratio is independent of size is not necessary in order to solve for the hail size parameters. In fact, if the dependence of the axis ratio on diameter can be calibrated from measurements, and it is found to be similar for certain storm types, then $K_{D P}$ and $Z_{D R}$ may be used to solve for an additional parameter, like the liquid-ice ratio of hailstones or their maximum diameter.

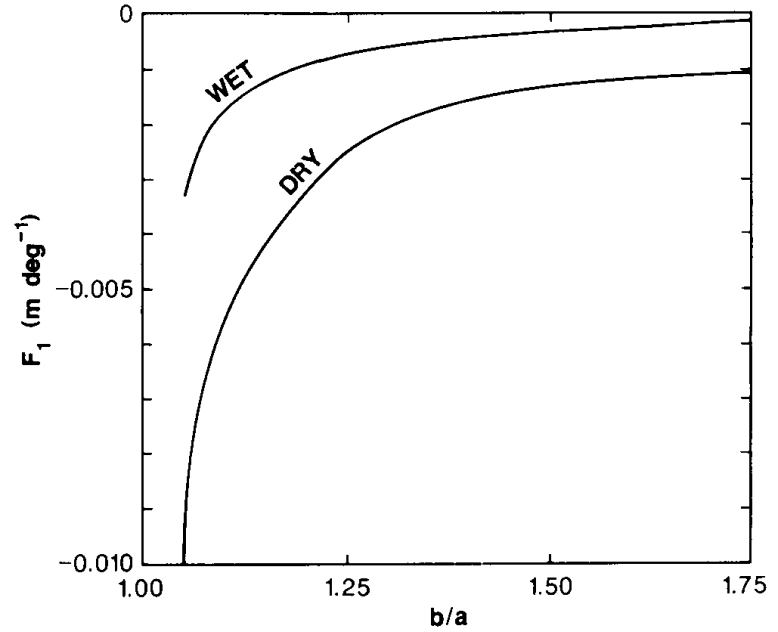

(a)

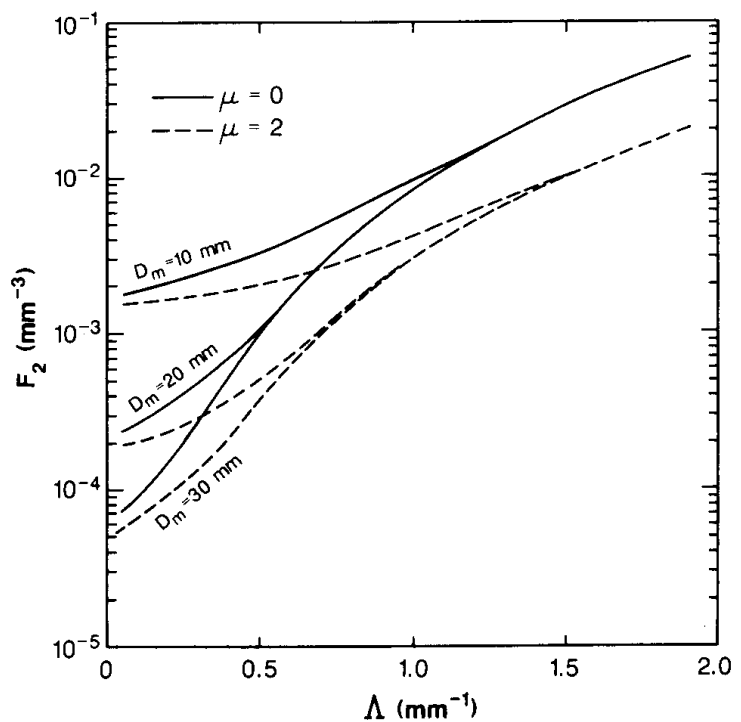

(b)

Fig. 3. Dependence of functions $F_{1}$ and $F_{2}$ on hail parameters: (a) $F_{1}$ versus the axis ratio $b / a$ for dry and 10-percent wet hail of density 0.5 $\mathrm{g} / \mathrm{kg}$. (b) $F_{2}$ versus $\Lambda$ and $D_{m}$ as parameters for exponential and gamma distributions.

\section{Radar MEASUREMENTS}

The data presented in this study are from rain showers on June 4 and 10, 1986, and from a hail storm on June 2, 1985. They were collected with a radar having a $10-\mathrm{cm}$ wavelength and the capability of switching polarization from pulse-to-pulse. Simultaneous collection of time series data from 16 consecutive range locations was possible and the spacing could be adjusted in powers of 2 , from 1 to $16 \mu \mathrm{s}$. 512 alternately polarized samples from each range location were processed, as explained in Section II. Theoretically computed standard error of $K_{D P}$ estimates is about $0.6 \mathrm{deg} \mathrm{km} \mathrm{km}^{-1}$ when the resolution is $1.2 \mathrm{~km}$ and 0.3 $\operatorname{deg~} \mathrm{km}^{-1}$ when the resolution is $2.4 \mathrm{~km} \mathrm{[6].} \mathrm{There} \mathrm{are} \mathrm{no}$ system induced biases in the $K_{D P}$ estimates, but in regions of weak reflectivities near strong cores, contamination by 

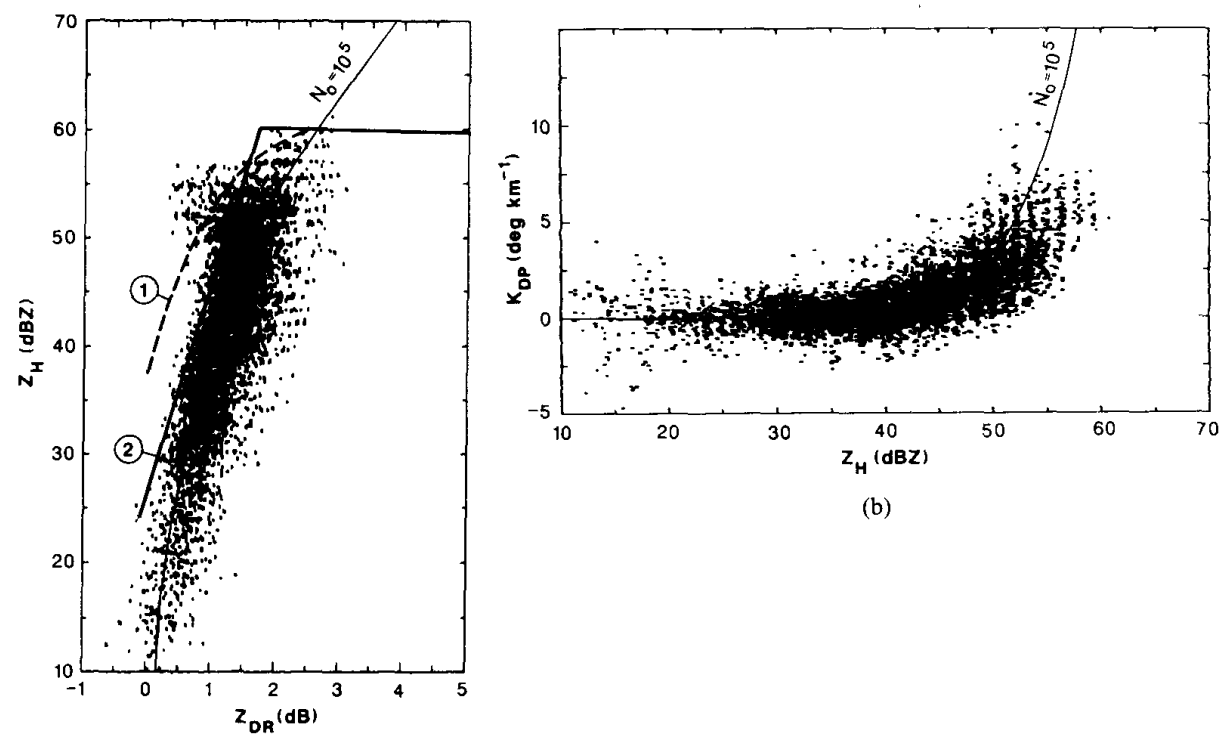

(b)

(a)

Fig. 4. Scatterograms for a rainfall on June 10,1986 , from the elevation of $1^{\circ}$, with a curve for $N_{0}=10^{5} \mathrm{~mm}^{-1} \mathrm{~m}^{-3}$ : (a) $Z_{H}-Z_{D R}$ and (b) $K_{D P}-Z_{H}$, and the rain boundaries, marked (1) from [20] and (2) from [21].

echoes through sidelobes is possible. This was not the case in the analyzed data. Standard errors of reflectivity estimates are better than $1 \mathrm{~dB}$, and biases are kept below 1 dB. Standard errors of differential reflectivity are less than $0.1 \mathrm{~dB}$, and there were no systematic biases [27].

In Fig. 4 results of the radar measurements on June 10, 1986 are shown. These were obtained from a sector of 30 degrees, and between the ranges of 37 and $63 \mathrm{~km}$, at an elevation of $1^{\circ}$. Polarization parameters were calculated from time series data that were spaced in range by $150 \mathrm{~m}$. For comparison, $Z_{H}-Z_{D R}$ boundaries for rain [20], [21] are marked, along with the calculated curve for $N_{0}=10^{5}$ $\mathrm{mm}^{-1} \mathrm{~m}^{-3}$ of an exponential DSD with $D_{m}=8 \mathrm{~mm}$. Scatterograms show that the majority of $Z_{H}-Z_{D R}$ data points (all measured at $1^{\circ}$ elevation) lie in the range considered typical for rain. Some data points show $Z_{D R}$ close to zero at relatively high reflectivity, and hence outside the rain boundary, presumably indicating the presence of partially melted hailstones, or drops with ice cores (the freezing level was at a $4-\mathrm{km}$ height). $Z_{H}-Z_{D R}$ points indicate large variability in DSD (see also Fig. 2), with $N_{0}$ from $10^{3}$ to $10^{6} \mathrm{~mm}^{-1} \mathrm{~m}^{-3}$, and $\Lambda$ larger than $2 \mathrm{~mm}^{-1}$ $\left(Z_{D R}\right.$ values are relatively small, generally below $2.5 \mathrm{~dB}$ ). There is also a corresponding high variability in rainfall rate, from less than $1 \mathrm{~mm} \mathrm{~h}^{-1}$ (drizzles, where reflectivity is lower than $25 \mathrm{dBZ}$ ) to $200-300 \mathrm{~mm} \mathrm{~h}^{-1}$ and over, corresponding to high reflectivity close to the rain boundary. $K_{D P}$ data (Fig. $4(\mathrm{~b})$ ) are correlated with $Z_{H}$ as expected for raindrops. There are, however, many points with negative $K_{D P}$ values, which may be partially explained in terms of statistical uncertainty of $K_{D P}$ measurements. On the other hand, $K_{D P}$ values corresponding to higher reflectivities are consistently lower than expected. For instance, for $Z_{H}=55 \mathrm{dBZ}$ and $Z_{D R}=1.5$ to $2 \mathrm{~dB}, K_{D P}$ is expected to be over $10 \mathrm{deg} \mathrm{km}^{-1}$. The measured values, 7 to $8 \mathrm{deg} \mathrm{km}^{-1}$, would fit the theoretical ones (for the same $Z_{H}$ ) if $Z_{D R}$ were 3.5 to $4 \mathrm{~dB}$. Assuming an $N_{0}$ of $10^{4}$ to $10^{5} \mathrm{~m}^{-3} \mathrm{~mm}^{-1}$, this conclusion is nearly independent of $D_{m}$ (see Fig. 1(c)) and also holds for the examined gamma distributions (Fig. 1(d)). Perhaps some less realistic DSD would fit these data, or a simpler explanation is possible? A source for relatively low $K_{D P}$ values could be a mixed population of rain and ice pellets.

To illustrate this point, data from another rain case (June $4,1986)$ are presented in Fig. 5. These data were obtained from a stationary beam above Norman and are from ranges $38-40 \mathrm{~km}$ and at an elevation of $0.7^{\circ}$. At that elevation, reflectivity is relatively high, with only a few points below $45 \mathrm{dBZ}$. Only rain was observed on the ground. About 70 percent of the $Z_{H}, Z_{D R}$ data are within the rain boundary and have a rain rate in excess of $100 \mathrm{~mm} \mathrm{~h}^{-1}$. As in the previous case, there are no negative $Z_{D R}$ values in this high reflectivity region, although some are rather low. $K_{D P}$ data are positive, as is expected for rain, with only a very few scattered data points below zero. This may then represent pure rainfall; in effect, no observations of ice phase hydrometeors have been reported. A more careful examination of $K_{D P}$ data shows that, whereas the mean $N_{0}$ values based on $Z_{H}-Z_{D R}$ data and exponential distribution are about $10^{5} \mathrm{~mm}^{-1} \mathrm{~m}^{-3}$, from $Z_{H}-K_{D P}$ measurements, one gets $N_{0}$ closer to $10^{4} \mathrm{~mm}^{-1} \mathrm{~m}^{-3}$. Most $Z_{H}-K_{D P}$ data points (those that correspond to $Z_{H}-Z_{D R}$ data within the rain boundary, and the others outside) are within the range of $K_{D P}=1$ to $4 \mathrm{deg} \mathrm{km}{ }^{-1}$, for $Z_{H}=45$ to $55 \mathrm{dBZ}$. However, $Z_{H}-K_{D P}$ data points corresponding to $Z_{H}-Z_{D R}$ within the rain boundary (below $60 \mathrm{dBZ}$ ) include in addition, some higher $K_{D P}$ values. This group has more data points of medium reflectivity values than the other one, which cor- 


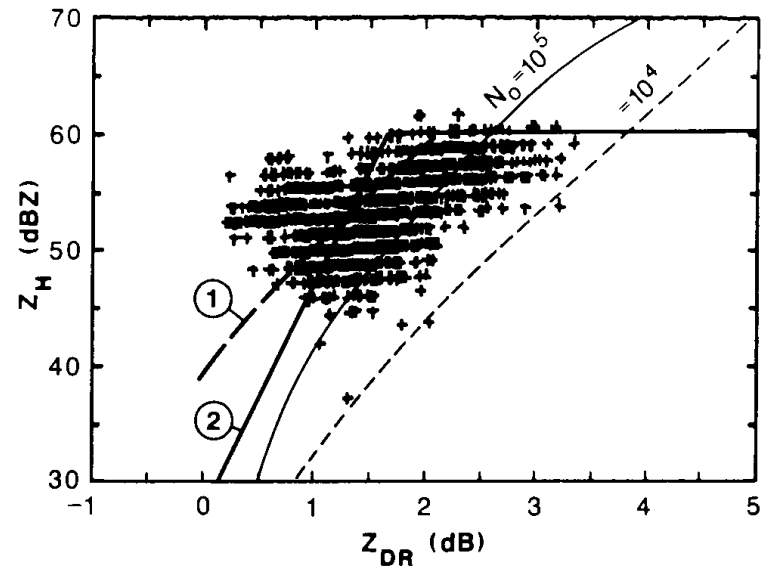

(a)

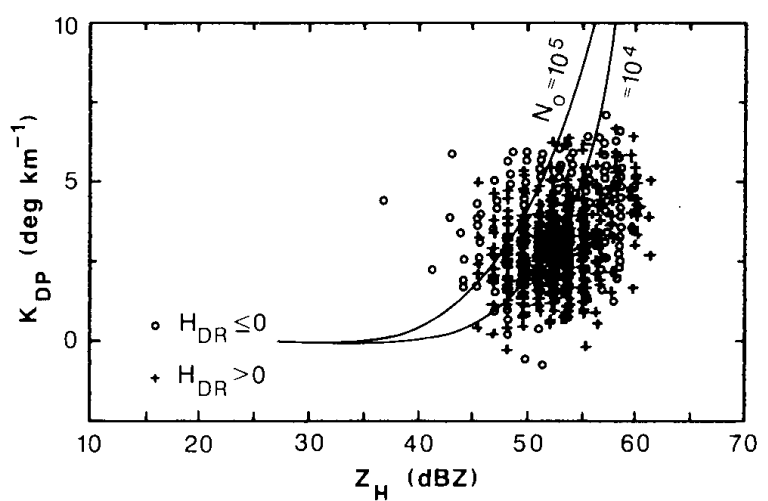

(b)

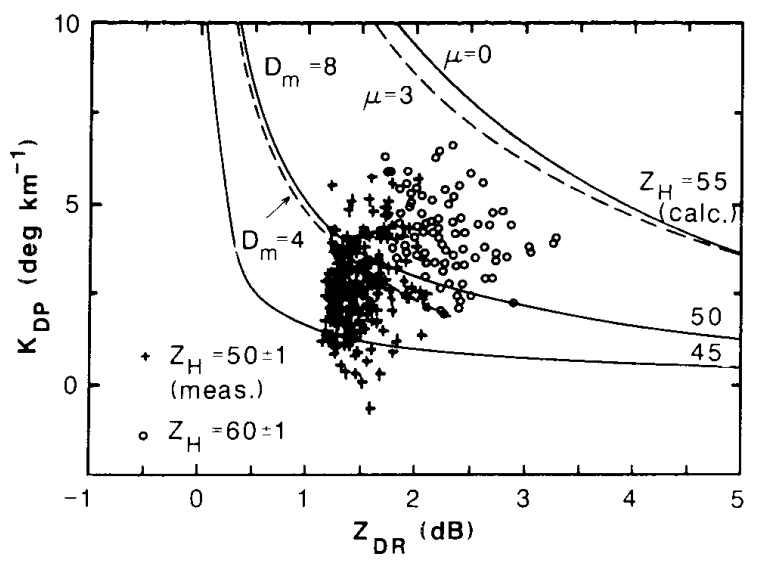

(c)

Fig. 5. Scatterograms for a storm on June 4, 1986, from the elevation of $0.7^{\circ}$ : (a) $Z_{H}-Z_{D R}$ data are presented with curves of equal $N_{0}$ (in $\mathrm{mm}^{-1}$ $\mathrm{m}^{-3}$ ) and the rain boundaries (1) [20] and (2) [21]. (b) $K_{D P}-Z_{H}$ data subdivided into groups within the rain boundary and outside $\left(H_{D R}>0\right)$, with theoretical curves for two $N_{0}$ 's. (c) $K_{D P}-Z_{D R}$ data for $H_{D R}<0$. Circles indicate data with reflectivities between 59 and 61 , whereas crosses are for data with $Z_{H}$ between 49 and $51 \mathrm{dBZ}$. Theoretical graphs for exponential DSD $\left(D_{m}=8 \mathrm{~mm}\right)$ at reflectivities of 45,50 , and 55 $\mathrm{dBZ}$, exponential DSD $\left(D_{m}=4 \mathrm{~mm}\right)$ at $50 \mathrm{dBZ}$, and gamma DSD $\left(D_{m}\right.$ $=8 \mathrm{~mm}$ and $\mu=3$ ), at $55 \mathrm{dBZ}$ are superposed. responds to $Z_{H}-Z_{D R}$ data outside the rain regime. These data points are further examined in Fig. 5(c) via a $K_{D P^{-}}$ $Z_{D R}$ scatterogram. Data are grouped into two categories. One set contains all $K_{D P}-Z_{D R}$ pairs with $Z_{H}$ between 49 and $51 \mathrm{dBZ}$; the other set has points with $Z_{H}$ between 59 and $61 \mathrm{dBZ}$; both are within the rain boundary. The correlation of $K_{D P}$ with $Z_{H}$ and with $Z_{D R}$ may clearly be seen. A comparison with theoretical $K_{D P}-Z_{D R}$ values for an exponential DSD with $D_{m}=8 \mathrm{~mm}$ shows that such distribution is possible for data corresponding to $Z_{H}=50 \mathrm{dBZ}$, although the theoretical $Z_{H}$ values seem to be biased high by 2 to $3 \mathrm{dBZ}$, compared to the mean measured values. A DSD with $D_{m}=4 \mathrm{~mm}$ yields a better fit to the measured data. Thus, the addition of $K_{D P}$ measurements points toward a narrower DSD, which is consistent with other variables, but cannot be independently verified. The data with $Z_{H}=60 \mathrm{dBZ}$ (and others above $55 \mathrm{dBZ}$, not included in Fig. 5(c)), show $K_{D P}$ lower by $5 \mathrm{deg} \mathrm{km}^{-1}$, or $Z_{D R}$ lower by $2 \mathrm{~dB}$ than expected for rain (see Fig. 2). If gamma distribution is considered, $K_{D P}$ data would be lower by $0.5-1 \mathrm{deg} \mathrm{km}{ }^{-1}$ only. The consequence is that the regions of higher reflectivity values, though within the rain boundary according to $Z_{H}-Z_{D R}$ measurements, may not represent rain of either exponential or gamma distribution. Rain may be mixed with other hydrometeors, whose scattering properties are more isotropic than those of rain alone.

Results of the measurements in rainfall regimes are therefore consistent with theoretical calculations, pointing out the possibility that some of the rain, mainly in the range of high reflectivity, could be mixed with ice phase hydrometeors. Data in these cases show positive $Z_{D R}$. This is not so in the case of June 2, 1985, as is shown in Figs. 6 and 7 , where some high reflectivity data have substantial negative $Z_{D R}$ from -1 to $-2.2 \mathrm{~dB}$ at a height of 0.2 $\mathrm{km}$. Hail signal values, based on criteria of Aydin et al. [21], are over $30 \mathrm{~dB}$ (Fig. 7). Hail was verified on the ground by the University of Oklahoma observers, who saw hailstones $2-3 \mathrm{~cm}$ in diameter and a mixture of hail and rain in several locations. $K_{D P}$ data also show some negative values, generally -0.5 to $-3 \mathrm{deg} \mathrm{km}^{-1}$, associated with negative $Z_{D R}$ and high $H_{D R}$. There are also some data points of negative $Z_{D R}$, with positive $K_{D P}$, and vice versa. Positive $Z_{D R}$ values are less than $1.7 \mathrm{~dB}$. This is lower than expected for rain in regions of high reflectivity (above $50 \mathrm{dBZ}$ ). When related to $Z_{H}$ (Fig. 6(a)), they imply $N_{0}$ of over $10^{6} \mathrm{~mm}^{-1} \mathrm{~m}^{-3}$. On the other hand, $K_{D P}-Z_{H}$ pairs require rather low $N_{0}$ of only $100 \mathrm{~mm}^{-1} \mathrm{~m}^{-3}$, if interpreted as originating from rain. Therefore, it appears that the dominant hydrometeors were in the form of hail.

This case is rather exceptional with regard to $Z_{D R}$ data reported by other investigators, which are generally close to zero for hail [3]. The largest negative $Z_{D R}$ value reported so far was an isolated point of $-1.4 \mathrm{~dB}$ [28]. The measured negative $Z_{D R}$ and $K_{D P}$ of the June 2 storm could be attributed to scattering from small hail falling, on the average, with major axis vertically oriented. Another pos- 

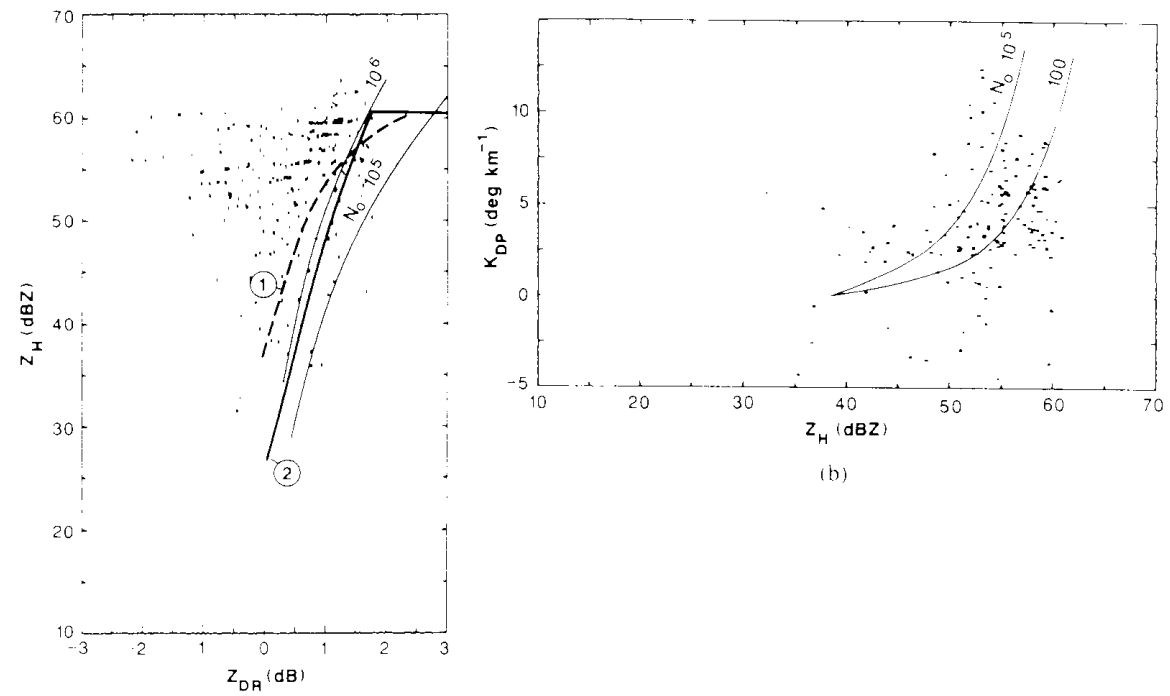

(i)

Fig. 6. Scatterograms from a hail storm on June 2. 1985: the elevation is $0.2:$ (a) $Z_{f 1}-Z_{p R}$ with the rain boundaries (1) 1201 and $(2) 1211$ and curves of constant $v_{0}$ values. (b) $K_{t p p}-Z_{p t}$ with two curves of constant $N_{0}$ superposed.
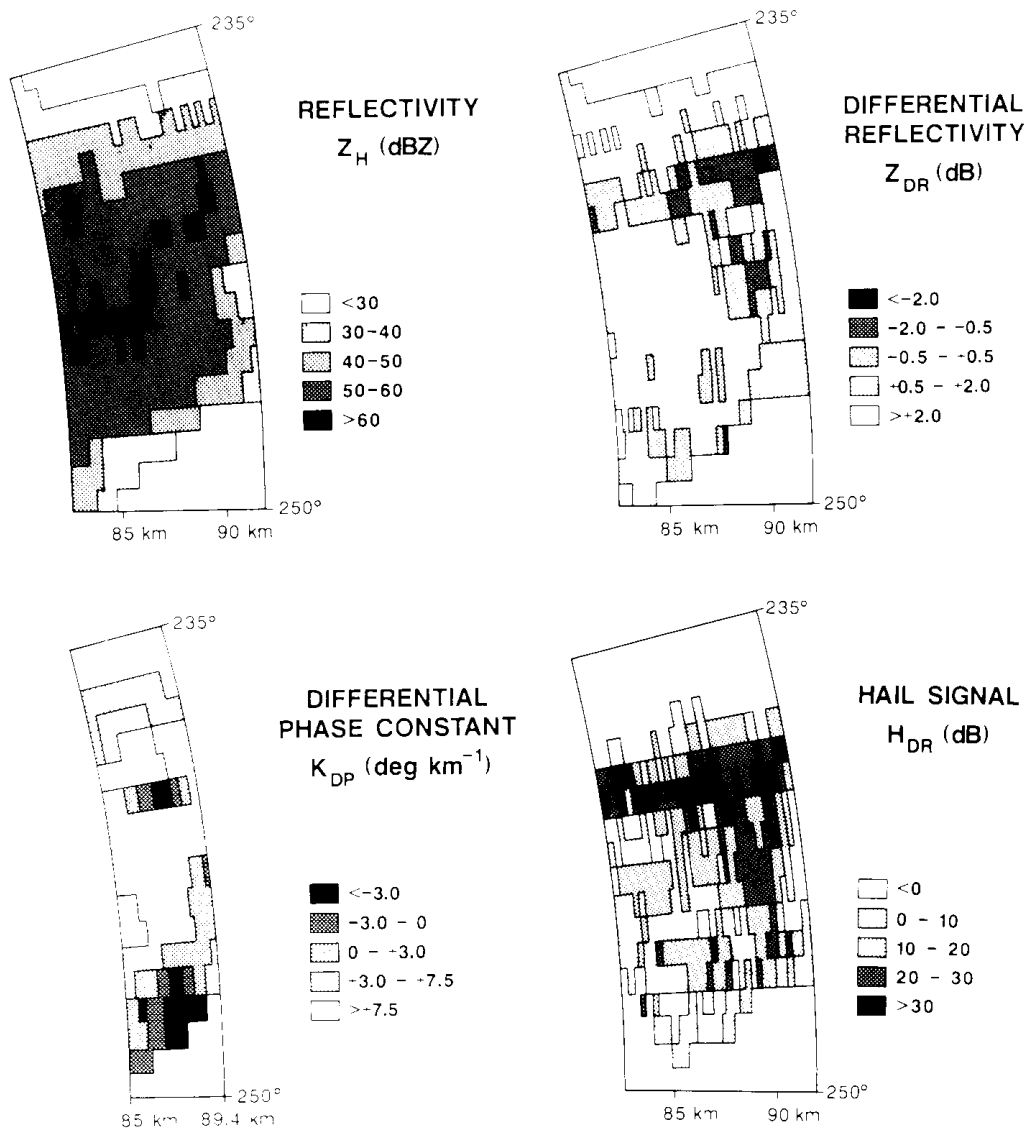

Fig. 7. Cross sections of $Z_{H i} . Z_{D R}$. and $K_{D P}$, and calculated $H_{D R}$ values following [21], of a hail storm on June 2 . 1985 . Beam center is at $0.2^{\circ}$ in elevation, but the lower part of the beam is blocked. Azimuthal limits of these sectors are $235^{\circ}-250^{\circ}$, the range from the radar is $81-94 \mathrm{~km}$. and only data that have reflectivities larger than $30 \mathrm{~dB}$. are displayed. 
sibility could be that contributing hailstones are, on the average, horizontally oriented, but the dominant sizes are such that Mie scattering produces stronger reflection at vertical polarization [24], [29]. Because of simplicity we consider further only the first possibility. In that case, one may apply the method just presented to get some quantitative information on the two parameter hail size distribution. The June 2 hail storm had high $Z_{H}$ values of 60 $65 \mathrm{dBZ} . Z_{D R}$ of -1 to $-2 \mathrm{~dB}$ indicate mean axis ratio of $0.77-0.88$ for wet hail, similar to observations in a Colorado storm [9]. Because high reflectivity and negative $Z_{D R}$ extend through a depth of $10 \mathrm{~km}$, contamination via antenna sidelobes can be excluded. A nearby ridge that partially blocks the beam also eliminates all ground clutter from the range beyond $50 \mathrm{~km}$. Calculated $\Lambda$ ranges from 0.05 to $1.1 \mathrm{~mm}^{-1}$, depending on $K_{D P}$ (which ranges from -0.5 to $-3 \mathrm{deg} \mathrm{km}^{-1}$ ), the distribution (exponential or gamma), and the maximum assumed size, 10 to 30 mm (see Fig. 3). The estimated $N_{0}$ are typically between 2 and $300 \mathrm{~mm}^{-1} \mathrm{~m}^{-3}$, although lower values, 0.01 , for higher $K_{D P}$, and higher, 600 , for dry hail, were also calculated. The classic measurements by Douglas [30] had $\Lambda=0.3 \mathrm{~mm}^{-1}$ and $N_{0}=3.1 \mathrm{~mm}^{-1} \mathrm{~m}^{-3}$, for hail size over $5 \mathrm{~cm}$. Other studies [31] suggest $\Lambda=0.4 \mathrm{~mm}^{-1}$ and $N_{0}=12 \mathrm{~mm}^{-1} \mathrm{~m}^{-3}$. Hail study of an Oklahoma storm [32] indicates gamma distributions with $\Lambda=0.05-0.1$ $\mathrm{mm}^{-1}$ and $N_{0}=0.005-0.01 \mathrm{~mm}^{-1} \mathrm{~m}^{-3}$. A wider range of $\Lambda$ and $N_{0}$ has been measured for wet hail [33]: $\Lambda=$ 0.1-1.5 mm $\mathrm{mm}^{-1}$ and $N_{0}=0.01-3000 \mathrm{~mm}^{-1} \mathrm{~m}^{-3}$. Although our deduced values are within the range of previous measurements, it is premature to draw positive inferences. Verification of the falling characteristics of the hail, and combined radar and in situ measurements, are needed before a conclusion can be made on the usefulness of this method for hail size estimation.

\section{Conclusions}

Measurements of the differential phase constant $K_{D P}$ are examined in conjunction with the reflectivity and differential reflectivity with the aim of obtaining additional information on rain and hail characteristics. Although more effort is needed to improve $K_{D P}$ measurements, this parameter adds information that is unavailable from dualpolarization measurements of reflectivity alone. The range resolution of $K_{D P}$ estimates is lower than the inherent resolution possible, but it is compatible with the operational $1-\mathrm{km}$ resolution of reflectivity estimates. It is shown that $K_{D P}$ may be used to support $Z_{D R}$ measurements with regard to the hydrometeor phase, or alternately to detect the presence of hydrometeors, other than raindrops, when $Z_{H}$ and $Z_{D R}$ data are within the range typical for rain. Calculations based on Rayleigh scattering suggest that with $K_{D P}$ measurements, one could solve for a third parameter of DSD, which may be either $D_{m}$, when exponential distribution is assumed, or $\mu$, in the case of a gamma distribution. Assuming that small hailstones fall with their major axes aligned vertically, the use of $K_{D P}$ together with $Z_{H}$ and $Z_{D R}$ may enable remote quantitative estimation of their size distributions. We presented data from one hailstorm in which a predominent orientation of hailstones resulted in negative $Z_{D R}$ and $K_{D P}$. Observed negative $Z_{D R}$ (minimum $-2.2 \mathrm{~dB}$ ) and $K_{D P}\left(\right.$ minimum $-3 \mathrm{deg} \mathrm{km}^{-1}$ ) in regions of high reflectivity (over $55 \mathrm{dBZ}$ ) suggest that these hailstones in the freefall could, on the average, be vertically oriented. However, horizontal orientation (like raindrops) of certain hail sizes may, in the Mie regime of scattering, produce similar results [24]. This possibility needs further exploration. The effectiveness of $K_{D P}$ measurements increases with increasing reflectivity, as the standard error of the measurements is constant (less than $0.6 \mathrm{deg} \mathrm{km}^{-1}$ ) and $K_{D P}$ increases exponentially with $Z_{H}$. $K_{D P}$ may principally be used for rain rate estimation when reflectivity is larger than $40 \mathrm{dBZ}$. Furthermore, our study shows that the $K_{D P}$ measurements may be used to identify mixed phase regimes, for which $Z_{H}$ and $Z_{D R}$ are insufficient or ambiguous. Although independent confirmation, using in situ measurements, is needed to positively identify the type of precipitation above the ground, polarimetric measurements in storms of the Great Plains imply that hydrometeors of mixed phase are often present aloft.

\section{ACKNOWLEDGMENT}

The authors appreciate efforts of the NSSL engineering staff; led by D. Sirmans and J. Carter, who made the modifications to the radar. B. Bumgarner and Y. Gal-Chen provided guidance in the use of computer software. $M$. Schmidt and C. Clark operated the radar. Dr. Sachidananda's perceptive suggestions and pioneering efforts in studies of $K_{D P}$ were most inspiring. Dr. Ziegler, Dr. Balakrishnan, and Dr. Maddox gave us valuable advice. Y. Gal-Chen provided assistance in the use of computing routines, C. Holder typed the manuscript, and J. Kimpel produced the figures. Dr. Steinhorn was an NRC Postdoctoral Fellow during the course of this work.

\section{REFERENCES}

[1] T. A. Seliga and V. N. Bringi, "Potential use of radar reflectivity at orthogonal polarizations for measuring precipitation,"' J. Appl. Meteorol., vol. 15, pp. 69-76, 1976.

[2] M. P. M. Hall, S. M. Cherry, J. W. F. Goddard, and G.R. Kennedy, "Rain drop size and rainfall rate measured by dual-polarization radar,'” Nature, vol. 85, pp. 195-198, 1980.

[3] V. N. Bringi, T. A. Seliga, and K. Aydin, "Hail detection with a differential reflectivity radar," Science, vol. 225 , pp. 1145-1157, 1984

[4] P. A. Watson and M. Arbabi, "Rainfall cross polarization at microwave frequencies," Proc. IEE, vol. 120, no. 4, pp. 413-418, 1973.

[5] A. R. Jameson, "Microphysical interpretation of multiparameter radar measurements in rain: Part III: Interpretation and measurements of differential phase shift between orthogonal linear polarizations," J. Atmos. Sci., pp. 607-616, 1985.

[6] M. Sachidananda and D. S. Zmic', 'Differential propagation phase shift and rainfall rate estimation," Radio Sci., vol. 21, pp. 235-247, 1986.

[7] - , "Rain rate estimates from differential polarization measurements,' J. Atmos. Oceanic Tech., vol. 4, no. 3, pp. 588-598, 1987.

[8] H. C. Van de Hulst, Light Scattering by Small Particles. New York: Wiley, 1975

[9] R. J. Matson and A. W. Huggins, "The direct measurement of the sizes, shapes and kinematics of falling hailstones," J. Atmos. Sci., vol. 37, pp. 1107-1125, 1980 . 
[10] T. Oguchi, "Electromagnetic wave propagation and scattering in rain and other hydrometeors,"' Proc. IEEE, vol. 71, pp. 1029-1078, 1983.

[11] R. J. Doviak and D.S. Zrnic', Doppler Radar and Weather Obser vations, New York: Academic, 1984.

[12] D. Sirmans and J. T. Dooley, "A numerical comparison of three differential reflectivity estimators,"' in Proc. 23rd Conf. Radar Meteorol. (Snow-mass, CO), pp. 189-192, 1986.

[13] A. W. Green, "An approximation for the shape of large raindrops," J. Appl. Meteorol., vol. 14, pp. 1578-1583, 1975.

[14] H. R. Prupacher and R. L. Pitter, "A semi-empirical determination of the shape of cloud and raindrops," J. Atmos. Sci., vol. 28, pp. $86-94,1971$.

[15] J. S. Marshall and W. Palmer, "The distribution of raindrops with size," J. Meteorol, vol. 5, pp. 165-166, 1948.

[16] A. Waldvogel, "The $N_{0}$ jump of raindrop spectra," J. Atmos. Sci., vol. 31, no. 4, pp. 1067-1078.

[17] C. W. Ulbrich, "Natural variations in the analytical form of the raindrop size distribution," J. Clim. Appl. Meteorol., vol. 22, pp. 1764$1775,1983$.

[18] _ - " "The effects of drop size distribution truncation on rainfall integral parameters and empirical relations," in Proc. 22nd Conf. Radar Meteorol. (Zurich, Switzerland), pp. 332-337, 1984.

[19] N. Balakrishnan, D. S. Zrnic, J. Goldhirsh, and J. Rowland, "Comparison of simulated rain rate accuracies from disdrometer data employing polarimetric radar algorithms," submitted to J. Atmos. Oceanic Tech., 1988.

[20] M. J. Leitao and P. A. Watson, "Application of dual-linearly polarized radar data to prediction of microwave path attenuation at $10-30$ GHZ," Radio Sci., vol. 19, pp. 209-221, 1984.

[21] K. Aydin, T. A. Seliga, and V. Balaji, "Remote sensing of hail with a dual linear polarization radar,' J. Clim. Appl. Meteorol., vol. 25, pp. 1475-1484. 1986.

[22] C. A. Knight and N. C. Knight, "The falling behavior of hailstones," J. Atmos. Sci., vol. 27, pp. 672-681, 1970.

[23] P. R. Kry and R. List, "Angular motions of freely falling spheroidal hailstone models," Phys. Fluids, vol. 17, pp. 1093-1102, 1974.

[24] D. R. Longtin, C. F. Bohren, and L. J. Battan, "Radar back scattering by large, oblate spongy ice spheroids," J. Atmos. Oceanic Tech., vol. 4, no. 3, pp. 355-358, 1987.

[25] B. L. Barge and G. A. Isaac, "The shape of Alberta hailstones,"' $J$. de Rech. Atmos., vol. 7, pp. 11-20, 1973.

[26] N. C. Knight, "Hailstone shape factor and its relation to radar inter pretation of hail,' 'J. Clim. Appl. Meteorol., vol. 25, pp. 1956-1958, 1986.

[27] M. Sachidananda and D. S. Zrnic', ' $Z_{D R}$ measurement consideration for a fast scan capability rader," Radio Sci., vol. 20, no. 4, pp. $907-$ $922,1985$.

[28] A. J. Illingworth, J. W. F. Goddard, and S. M. Cherry, "Polarization radar studies of precipitation development in convective storms," Quart. J. Roy. Meteorol. Soc., vol. 113, no. 476, pp. 469-489, 1987.

[29] T. A. Seliga and V. N. Bringi, "Differential reflectivity and differential phase shift: Applications in radar meteorology," Radio Sci., vol. 13, no. 2, pp. 271-275, 1978 .

[30] R. H. Douglas, "Size distribution of Alberta hail samples," Sci. Rep. MW-36, Stormy Weather Research Group, McGill Univ., Montreal, Sci. Rep. MW-36, pp. 55-70A, 1963.

[31] B. Federer and A. Waldvogel, "Hail and raindrop size distributions from a Swiss multicell storm," J. Appl. Meteorol., vol. 14, pp. $91-$ 97, 1975.
[32] C. L. Ziegler, P. S. Ray, and N. C. Knight, "Hail growth in an Oklahoma multicell storm,"'J. Atmos. Sci., vol. 40, pp. 1768-1791, 1983.

[33] L. Cheng and M. English, "Hailstone concentration and size at the ground and at the melting layer," in Proc. Conf. Cloud Phys., A.M. S., Chicago, IL, pp. 423-426, 1982.

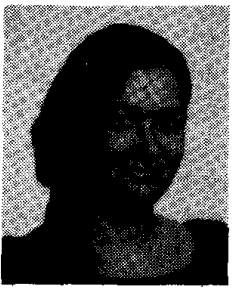

Ilana Steinhorn (M'87) was born and educated in Israel. She received the B.Sc. degree in physics and mathematics in 1972 and the M.Sc. degree in atmospheric sciences in 1974 from the Hebrew University of Jerusalem, where she was involved in research of microphysical aspects of clouds. She received the $\mathrm{Ph}$. D. degree in 1982 from the Weiz mann Institute of Science in Rehovot. Her thesis discussed hydrographical measurements of the water column of the hyper-saline Dead Sea, the physical properties of Dead Sea waters, and the mixing processes of the lake and adjacent solar ponds.

For two years, beginning in September 1985, she was a National Research Council (NRC) Associate at the National Severe Storms Laboratory in Norman, $\mathrm{OK}$, where here research interests included radar meteorology, emphasizing applications of polarimetric measurements to the identification of hydrometeors. At present she is a Research Associate in the Department of Electrical Engineering at Pennsylvania State University, University Park.

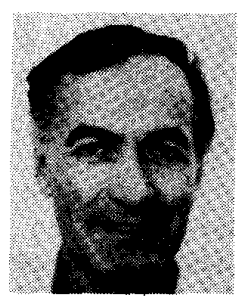

Dusan S. Zrnic (M'73-SM-77) received the B.S. degree in electrical engineering in 1965 from the University of Belgrade, and he received the M.S and the Ph.D. degrees in electrical engineering from the University of Illinois, Urbana.

Following employment as a research and teaching assistant with the Charged Particle Research Laboratory at the University of Illinois, he joined the Electrical Enginering Department of California State University, Northridge. He became. Associate Professor in 1974, and Professor in 1978. During the 1973-1974 academic year, he was a National Research Council Post-Doctoral Fellow at the NSSL, and from 1975-1976 he was on sabbatical at the same laboratory. He is now Chief of the Doppler Radar Group at the National Severe Storms Laboratory (NSSL), and Adjunct Professor of Electrical Engineering and Meteorology at the University of Oklahoma, Norman. His research experience includes circuit design, applied mathematics, magnetohydrodynamics, and signal-processing and radar systems. Recently he has spent considerable effort toward improvements of weather radar signal processing, advancements of polarimetric measurements and their interpretation, and development of algorithms for NEXRAD.

Dr. Zrnic has been a member of URSI Commissions C and F; he is also a member of AMS. Twice he has been awarded the best research paper award by the Environmental Research Laboratories. He is a corecipient of the IEEE 1988 Harry Diamond Memorial Award. 